
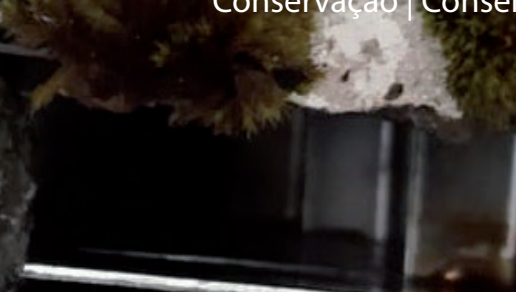

i

\title{
Inhibición del crecimiento de líquenes y musgos en elementos líticos usados en la construcción de fachadas de iglesias patrimoniales cusqueñas aplicando nanoburbujas de aire
}

\author{
Jhonny Wilfredo Valverde Flores, Carlos Guillermo Vargas Febres, Aris Cristobal Achahui \\ Alvarez, Danna Gabriela Rodriguez Salas
}

\begin{abstract}
Resumen: La investigación tuvo por objetivo evaluar la inhibición del crecimiento de líquenes y musgos presentes en los elementos líticos empleados en la construcción de fachadas de iglesias patrimoniales cusqueñas aplicando nanoburbujas de aire. Para dicho estudio se tomó una muestra de 20 elementos líticos provenientes de las canteras de origen para evitar experimentar en elementos pétreos de dichas iglesias. La longitud de cada muestra estuvo en el rango de 7 a $10 \mathrm{~cm}$ con presencia de agentes bióticos; la aplicación de nanoburbujas de aire se realizó por periodos de 10 minutos. Una vez concluido el experimento, las muestras fueron expuestas durante 04 semanas a factores climatológicos como lluvia, temperatura y presión atmosférica natural. En los resultados cualitativos se observa que las muestras con exposición de más de $10 \mathrm{~min}$. lograron desprendimientos y secado del agente biótico. De las fotografías analizadas se puede concluir que las nanoburbujas de aire siguen surtiendo efecto en periodos prolongados en las muestras analizadas hasta el punto de eliminar la patología mientras que en la muestra control se observa propagación y crecimiento de los mismos.
\end{abstract}

Palabras clave: nanoburbujas, patologías, elementos líticos, patrimonio, limpieza

\section{Inhibition of lichen and moss growth in lithic elements used in the construction of facades of Cusco heritage churches by applying air nanobubbles}

Abstract: The objective of the research was to evaluate the inhibition of lichen and moss growth present in the lithic elements used in the construction of facades of patrimonial Cusco churches by applying air nanobubbles. For this study, a sample of 20 lithic elements from the original quarries was taken to avoid experimenting with stone elements from said churches. The length of each sample was in the range of 7 to $10 \mathrm{~cm}$ with the presence of biotic agents; the application of air nanobubbles was carried out for periods of 10 minutes. Once the experiment was over, the samples were exposed for 04 weeks to climatic factors such as rain, temperature, and natural atmospheric pressure. In the qualitative results, it is observed that the samples with exposure of more than 10 min. they achieved detachments and drying of the biotic agent. From the analyzed photographs, it can be concluded that the air nanobubbles continue to affect over long periods in the analyzed samples to the point of eliminating the pathology, while in the control sample their propagation and growth are observed.

Keyword: nanobubbles, pathologies, lithic elements, heritage, cleanliness

\section{Inibição do crescimento de líquenes e musgos em elementos líticos usados na construção de fachadas de igrejas patrimoniais em Cusco pela aplicação de nanobolhas de ar}

Resumo: O objetivo da pesquisa foi avaliar a inibição do crescimento de líquenes e musgos presentes nos elementos líticos usados na construção de fachadas de igrejas patrimoniais de Cusco por meio da aplicação de nanobolhas de ar. Para este estudo, foi retirada uma amostra de 20 elementos líticos das pedreiras originais para evitar ensaios nos elementos de pedra das referidas igrejas. Cada amostra tinha entre 7 a $10 \mathrm{~cm}$ de comprimento com presença de agentes bióticos; a aplicação de nanobolhas de ar foi realizada por períodos de 10 minutos. Terminada a experiência, as amostras foram expostas por 4 semanas a fatores climáticos como chuva, temperatura e pressão atmosférica natural. Nos resultados qualitativos observou-se que as amostras com exposição superior a 10 
minutos apresentavam descolamentos e a secagem do agente biótico. Pelas fotografias analisadas concluiu-se que as nanobolhas de ar continuam a atuar por períodos prolongados nas amostras analisadas a ponto de eliminar a patologia, enquanto, na amostra de controlo, observa-se a propagação e crescimento de líquenes e musgo.

Palavras-chave: nanobolhas, patologias, elementos líticos, património, limpeza

\section{Introducción}

El rey Carlos I de España, en 1542, firmó en Barcelona las Ilamadas "Leyes Nuevas", conjunto legislativo que dispuso la creación del Virreinato del Perú. Posteriormente la ciudad del Cusco pasaría a ser una de las posesiones más valiosas de la Corona Española y, al mismo tiempo, el lugar donde se presentarían más estallidos revolucionarios en contra del Virreinato de Perú (Haring 1966).

Esta ciudad al ser en el pasado la capital del imperio de los Incas, es que por Real Cédula de Felipe II, se dispone la construcción de iglesias católicas donde se ubiquen templos o centros ceremoniales Incas. Por ello, la actual Plaza de Armas (Huaycaypata) fue elegida como el espacio destinado para la edificación de la más representativa infraestructura religiosa colonial. Una de las características fundamentales de tales iglesias fue la influencia del Manierismo empleando rocas como andesitas, dioritas y basaltos para las fachadas (Samanez 2019).

En 1733, se construye la Iglesia del Triunfo de Nuestra Señora, hoy Basílica Catedral del Cusco, que a lo largo de los años fue modificada hasta su actual imagen. El frontis o fachada de la Basílica Catedral, materia parcial de la presente investigación, fue construida entre los años 1658 y 1669 (Covarrubias 1958). Del mismo modo la iglesia de la Compañía de Jesús fue edificada a partir de 1571 por consentimiento del Virrey Toledo mediante la superposición al palacio del Inca Huayna Cápac. Las piedras con las que se construyó la iglesia, fueron transportadas de los propios muros del actual resto arqueológico de Sacsayhuamán (Covarrubias 1958). Luego del terremoto de 1650 la orden de los Jesuitas decide reconstruir la iglesia con estilo barroco utilizando la misma cantera de piedras tipo basalto rosado y andesita (Angles 1988).

El Centro Histórico de Cusco es reconocido como Patrimonio Cultural de la Humanidad por UNESCO en 1983, del mismo modo, la Constitución Política del Perú en su artículo 21, protege el patrimonio cultural y dentro de éste, especial consideración al patrimonio arquitectónico. Una de las mayores manifestaciones de dicho patrimonio, son las edificaciones religiosas coloniales, que fueron erigidas de material pétreo que provino de los propios muros Incas extraídos de las canteras de Huacoto, Rumicolca y Sacsayhuaman, siendo de tipo andesitas, calizas y dioritas. Con el pasar de los años, estas piedras comenzaron a manifestar patologías bióticas que afectan al elemento lítico llegando incluso a su destrucción y poniendo en peligro su conservación para las siguientes generaciones.

\section{Patologías del material pétreo}

De la revisión de la literatura especializada, se desprende que existen cuatro tipos de patologías (biológicas, químicas, físicas y mecánicas); cabe mencionar que el presente estudio se enfoca solamente a los agentes bióticos (líquenes y musgos) (Cabrera 1979) [tabla1].

Tabla 1.- Clasificación de patologías pétreas. (Broto 2006)

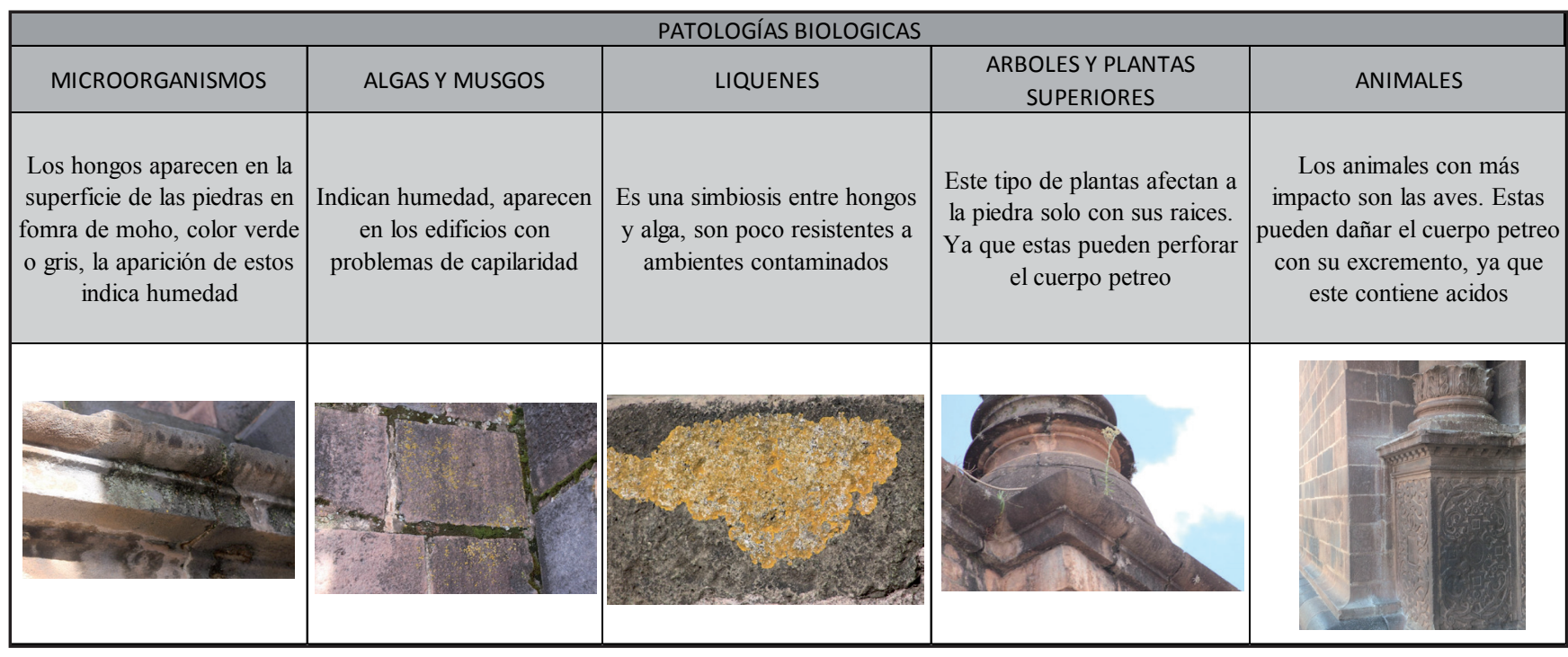


Cuando una roca se extrae de su cantera y se transforma para esculturas, tallados, pinturas, o construcciones se denomina elemento lítico y se expone a cambios en su estructura, ésto incluye el interior y exterior del elemento. Al estar expuesto, es afectado por diferentes factores o elementos que llegan a dañar su estructura. Los más comunes con alto grado de agresividad son: la humedad, sales solubles, organismos vivos, contaminantes atmosféricos. Sin embargo, para efectos de la investigación, se presenta una clasificación general de dichas alteraciones, daños y degradaciones que sufre el elemento lítico en las edificaciones arquitectónicas patrimoniales.

\section{Técnicas de limpieza de patologías pétreas}

Instituciones públicas, como el Ministerio de Cultura, a través de la Dirección Desconcentrada de Cultura (DDC), realizan trabajos de mantenimiento, conservación y restauración en las iglesias del centro histórico, utilizando técnicas como el empapetado desarrolladas décadas atrás y que consiste en cubrir el elemento lítico con un engrudo elaborado de papetas remojadas en agua destilada; sin embargo, los resultados de su aplicación no solucionan de manera efectiva y sostenible, sino que en muchos casos, como la limpieza con chorros de agua, terminan afectando irremediablemente al elemento lítico. Dichos trabajos resultan insuficientes ya que en la actualidad se pueden apreciar la existencia de patologías biológicas.

A pesar de contar con múltiples soluciones a las diversas patologías, son pocas y de alto costo en su aplicación. Por lo que plantear un sistema o tecnología alternativo que no ocasione alteraciones secundarias y que sea de fácil acceso económico para el tratamiento de dichas patologías se hace indispensable de investigación. Por lo que, en la presente investigación se muestran los resultados obtenidos de la aplicación de nanoburbujas mediante un sistema de neblina a elementos líticos extraídos de las mismas canteras empleadas en épocas prehispánicas, con lo que se evita la agresión y manipulación directa del elemento lítico patrimonial. Cabe resaltar que las nanoburbujas se han aplicado en el tratamiento de aguas domésticas e industriales (Abate y Valverde 2006; Valenzuela 2018).

Se establecen tres pasos de intervención de limpieza de patologías pétreas: (a) Pre consolidación, que se refiere a la aplicación de consolidantes comerciales elaborados por esteres etílicos del ácido silícico $\left(\mathrm{Si}(\mathrm{OEt})_{4}\right) \mathrm{n}$, que en reacción con la humedad, desarrolla un gel de sílice que produce una unión química al elemento lítico; (b) Tratamiento de juntas: para eliminar el polvo, hollín, materiales orgánicos ubicados en las juntas y morteros de elementos pétreos, siendo extraídos de manera manual con instrumentos y equipos artesanales; y (c) Limpieza; se aplica agua a baja presión y cepillos no adhesivos eliminando depósitos de polvo y excrementos de aves; luego se procede con la microabrasión de precisión como se aprecia en la siguiente lista (antes de técnicas de nano).
- Micro proyección de partículas: En general, muros de fábrica de sillería y molduras

- Limpieza Laser: Zonas ornamentadas o delicadas

- Limpieza química: Áreas con costra negra

- Tratamiento biocida: Zonas afectadas por colonizaciones biológicas, tales como cornisas, gárgolas y pináculos

Este tipo de soluciones, si bien es cierto ayudan a la conservación del patrimonio artístico y arquitectónico, sin embargo, se ha podido observar a lo largo de los años, que los resultados, solo son temporales; ya que las patologías vuelven a aparecer con el tiempo. Además, resultan en muchos casos destructivos al elemento lítico, ya que, al momento de realizar la limpieza, se llevan consigo no solo la patología, sino también partículas y en algunos casos fragmento del mismo.

De los antecedentes investigativos se analizó que los estados de deterioro pétreo en los elementos constructivos de la edificación militar "El Arsenal" (Arancibia, Romeo y Fernández 2015). La metodología empleada caracterizó la tipología de rocas empleadas, así como sus propiedades. Así mismo se estudiaron las alteraciones y su relación con los agentes medioambientales. En los resultados se encontraron 09 especies de porte arbóreo, arbustivas y herbáceas. Dentro de los signos de alteraciones se encontraron: Para Presencia de organismos vivos: Plantas superiores, algas, musgos, hongos y líquenes, excretas de animales.

En otro estudio, se plantean dos métodos de aplicación de nanopartículas, así el primero de ellos es el "bottom up" que se caracteriza por sintetizar nano partículas a través del depósito y desarrollo de cristales por medio de la aplicación líquida o vapor. El segundo de ellos se denomina "top down" teniendo como ejemplos los métodos de sol-gel o de spray pirolisis. Se propone el empleo del método de láser para la generación de nano partículas utilizadas en tratamientos de conservación. Finalmente concluye que las nano partículas funcionan como bactericidas y germicidas, a través de sustancias químicas que reaccionan con la luz solar o ultra violeta; el óxido de titanio $\left(\mathrm{TiO}_{2}\right)$ como material catalítico que, junto con el óxido de zinc, óxido de magnesio y nano partículas de paladio son opciones que se vienen estudiando en la actualidad (Gómez 2008).

Investigadores del grupo de Petrología Aplicada a la Conservación de Patrimonio del Instituto de Geo ciencias (CSIC-UCM) permite apreciar que la nanociencia contribuye a minimizar o reparar el daño en el material pétreo, se describe los tratamientos enfocados en nano partículas de hidróxido de calcio aplicados sobre rocas carbonáticas (dolomías) así como las características de consolidantes de la superficie pétrea. De esta manera, exponen al Nanorestore, producto que se aplica para la consolidación que mejoran las propiedades físicas e hídricas de los materiales tratados. Concluyen que las nano partículas por su tamaño pueden 
penetrar de mejor manera que otros productos, evitan la propagación y crecimiento de microroganismos; además mencionan la eficacia que resulta la aplicación de resonancias magnéticas nuclear, el microdrilling, rugosímetro, tomografía de rayos x computarizada o la microscopia Raman-Confocal son técnias contemporáneas extraídas de otras ciencias para la conservación del patrimonio arquitectónico y artístico (Gómez 2008).

Las patologías en tres tipos de piedras (areniscas, granito y esquistos), que se ubican en las fachadas exteriores de la Catedral de la Seu Vella de Lleida, Catedral de Santa María d'Urgell y el monasterio de Sant Pere de Rodes fueron estudiados (Gainza 2016). Dentro de los factores analizados en la metodología aplicada fueron la ubicación, orientación, el entorno y el clima; en un segundo paso, se registraron las patologías existentes mediante un mapping determinado por un análisis visual asumiendo las causas de los daños y proponiendo la aplicación de nano formulaciones para su tratamiento. El método de aplicación fue mediante un pulverizador con nano partículas consolidantes e hidrofugantes, aplicados a la superficie de las rocas utilizando sistemas de caudal regulable y sin presión, según la porosidad de la roca. Como resultado al que arriba la investigadora, es la propuesta de aplicación de nano partículas consolidantes e hidrófugos en la superficie de las rocas; sin embargo, deja claro que no se puede realizar la experimentación, debido la falta de acceso legal a los monumentos para su manipulación. Dentro de las conclusiones más sobresalientes, recomienda la investigación experimental para la comprobación de dichos productos.

\section{Metodología}

La investigación es aplicada a nivel descripto y explicativo. La población para la primera etapa estuvo conformada por los elementos arquitectónicos que componen la fachada de las iglesias, divididas en tres cuerpos (02 torres laterales y el cuerpo central); mientras que, para la segunda etapa, se considera como población a las rocas extraídas de las canteras donde se encuentran las rocas basalto, andesita, caliza y diorita. La unidad de análisis corresponde a los elementos líticos correspondientes a las fachadas de la Basílica Catedral y la iglesia de la Compañía de Jesús ubicadas en la plaza de armas de la ciudad de Cusco. El tamaño de la muestra para la primera etapa, el muestreo fue no probabilístico intencional debido a que no es factible la manipulación física de los elementos líticos, por ser considerados patrimonio cultural de la Humanidad por UNESCO. Para la segunda etapa, la muestra se determinó por 20 especímenes que divididas en número de 10 que contengan las dos patologías estudiadas (10 con líquenes, 10 con musgos), dichas muestras fueron extraídas de las canteras de Rumicolca, Huacoto y Sacsayhuman (canteras a las que pertenecen los elementos pétreos originales). Esto permitió la no agresión ni manipulación de elementos líticos originales de las fachadas estudiadas; sin embargo, al pertenecer a la misma cantera, sufre las mismas patologías descritas en el marco teórico.

\section{Etapas de la investigación:}

La presente investigación tuvo dos etapas:

1. Caracterización y descripción de las patologías encontradas en las fachadas de las iglesias patrimoniales.

2. Determinación de los efectos producidos por la aplicación de nanoburbujas de aire en cuatro patologías pétreas (líquenes, musgos)

\section{-Primera Etapa: Caracterización y descripción de las patologías encontradas en las fachadas de las iglesias patrimoniales}

Se procedió a la caracterización y descripción de las patologías en las fachadas de la Basílica Catedral, así como de la Iglesia de la Compañía de Jesús como unidades de análisis de nuestra investigación.

De los cuatro tipos de rocas analizadas, en promedio son atacadas por el $75 \%$ de patologías, y sólo el basalto se exenta de líquenes y la diorita de oxidación. También podemos inferir que las rocas pueden y son atacadas por dos a más patologías al mismo tiempo; la patología tipo liquen se presenta en el $100 \%$ de las muestras, con lo cual dichas patologías serán de mayor interés en el análisis de la aplicación de nanoburbujas de aire [tabla 2].

Tabla 2.-Resultados de la investigación de Nano productos, efectos y aplicaciones. (Gómez 2008)

\begin{tabular}{|c|c|c|}
\hline Nano productos & Efecto & Aplicación \\
\hline Hidróxidos de Ca, Mg & Eliminación de sales & Piedra, pinturas, madera, papel, morteros \\
\hline Hidróxidos de Sr & Limpieza de otros productos & Lienzos \\
\hline Hiero magnético (ferrita) & Consolidante & Pienzos \\
\hline Óxido de Silicio & Hidrofugante & Biocida \\
\hline Nano - óxidos & Diferentes yiedra & Dotural, ladrillos, madera, hormigón \\
\hline Óxidos de Mg, Zn, Ti, Pd; Plata & &
\end{tabular}


Se dibujaron las fachadas de ambas iglesias en software de dibujo con sus diferentes elementos arquitectónicos. Luego, utilizando las fichas de observación, se procesó y vaciaron los datos de tal manera que divididos en dos colores (verde y amarillo) diferentes se identificaron el tipo de patología presente en cada elemento arquitectónico de dichas fachadas. El resultado encontrado se presenta a través de un mapa de manchas que determina la ubicación y tipo de patología presente. [figura 1].

De lo observado en la Basílica Catedral, las patologías más frecuentes son las producidas por agentes bióticos y químicos, así se observaron, líquenes, musgos, costras negras, oxidación, eflorescencias y salinidad. De otro lado, los elementos con mayor daño fueron las partes superiores de los tres cuerpos, los cuales están más expuestos a los factores climatológicos, así lo pináculos y cornisas presentan alteraciones provocadas por líquenes y musgos, mientras que los sistemas de arquerías y muros donde se ubiquen las escorrentías, presentan daños químicos como costras negras, salinidad y oxidación. [figura 2].

Al igual que la fachada de la Basílica Catedral, las patologías presentes son las biológicas, químicas y físico mecánicas, siendo los líquenes, musgos, oxidación, costras negras, salinidad, fractura, disgregación y faltantes las más encontradas. En esta investigación solo se consideraron las patologías biológicas por líquenes y musgos.

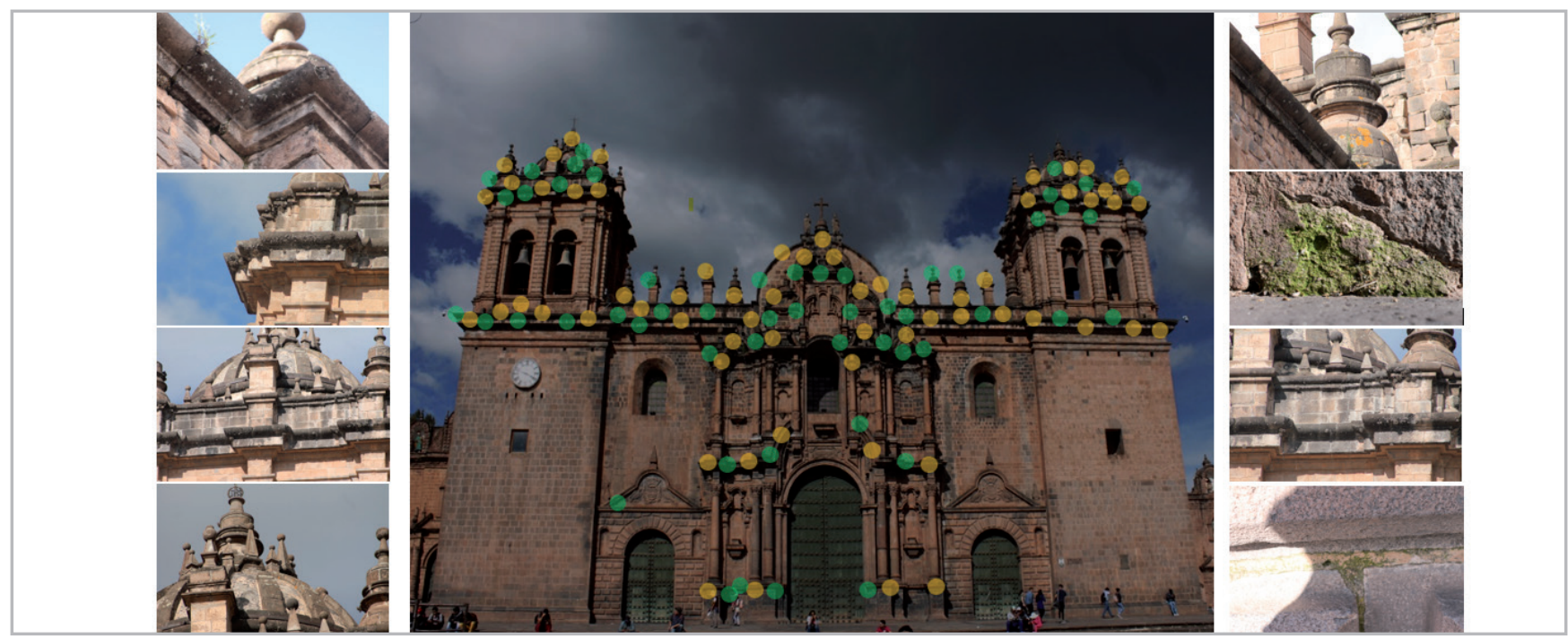

Figura 1.-Estado patológico de la Basílica Catedral de Cusco. Las manchas verdes representan presencia de musgo, y las amarillas de líquenes. Fuente: propia

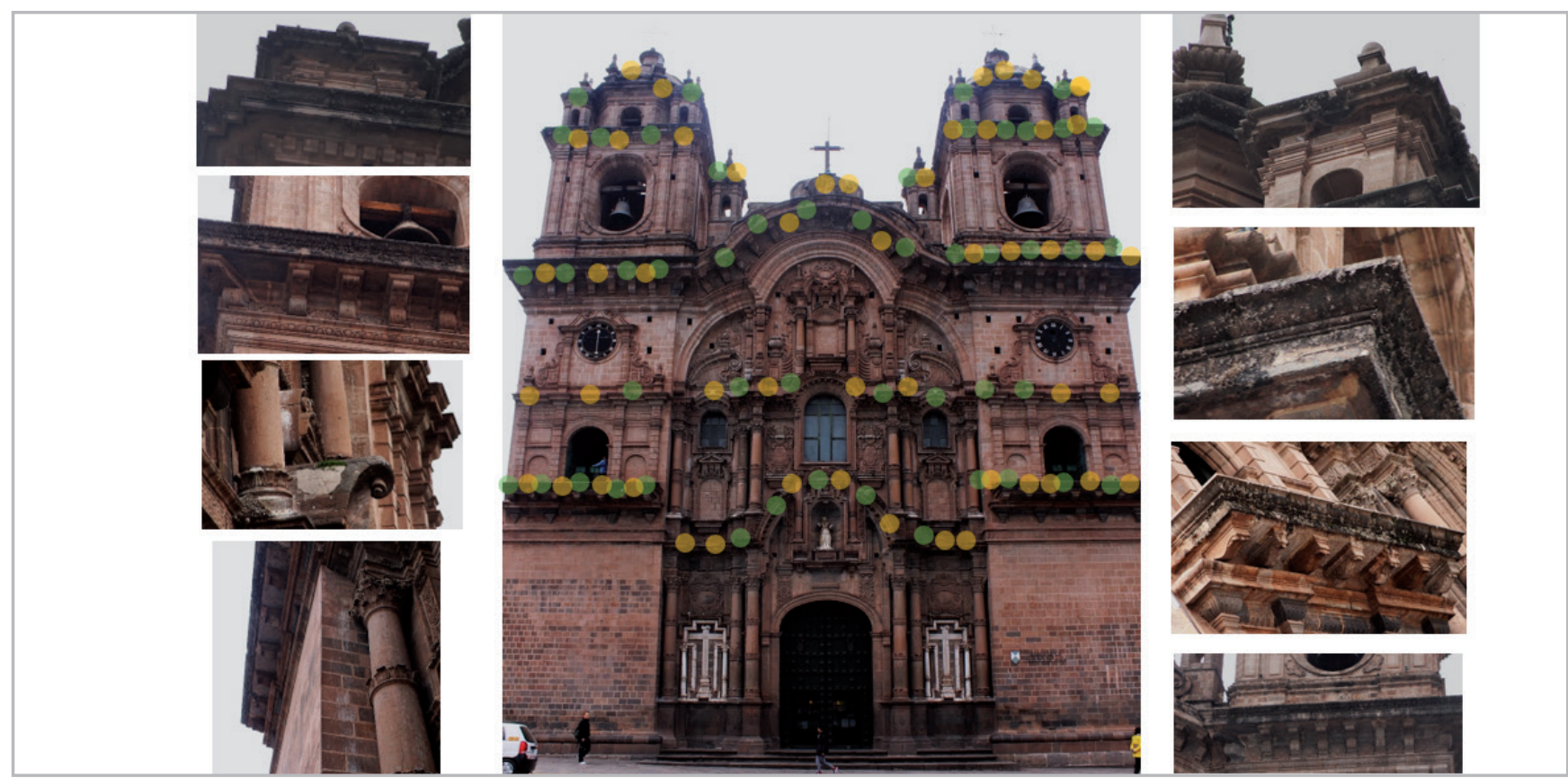

Figura 2.-Estado patológico de la Iglesia de la Compañía de Jesús, Las manchas verdes representan presencia de musgo, y las amarillas de líquenes. Fuente: propia 
-Segunda Etapa: Determinación de los efectos producidos por la aplicación de nanoburbujas de aire en dos patologías pétreas (Líquenes y musgos) [figura 3].

Entendida como la fase de experimentación de la investigación, se muestra el procedimiento experimental:

\section{-Procedimiento 1: Recolección de muestras}

Se recolectaron muestras en un número de 20 especímenes divididas en dos grupos de 10 que corresponden cada una a las siguientes patologías: Liquen (de LI-01 a LI-10), y Musgos (de MG-01 a MG-10).

-Procedimiento 2: Clasificación de muestras

Las muestras se sub dividen en dos grupos, uno a las cuales se aplicaron las nanoburbujas de aire en tres diferentes tiempos, y el otro grupo control al que no se le aplicaron las nanoburbujas.

-Procedimiento 3: Aplicación de nanoburbujas de aire

Se emplearon nanoburbujas de aire obtenidas del equipo patentado de generación de nano burbujas por el Dr. Jhonny Valverde Flores, de quién se tuvo la autorización respectiva. Como se muestra en la figura 4, dicho equipo se caracteriza por tener un espacio cerrado con un sistema de neblina de nanoburbujas de aire en su interior donde se introducen las diferentes muestras para su tratamiento.

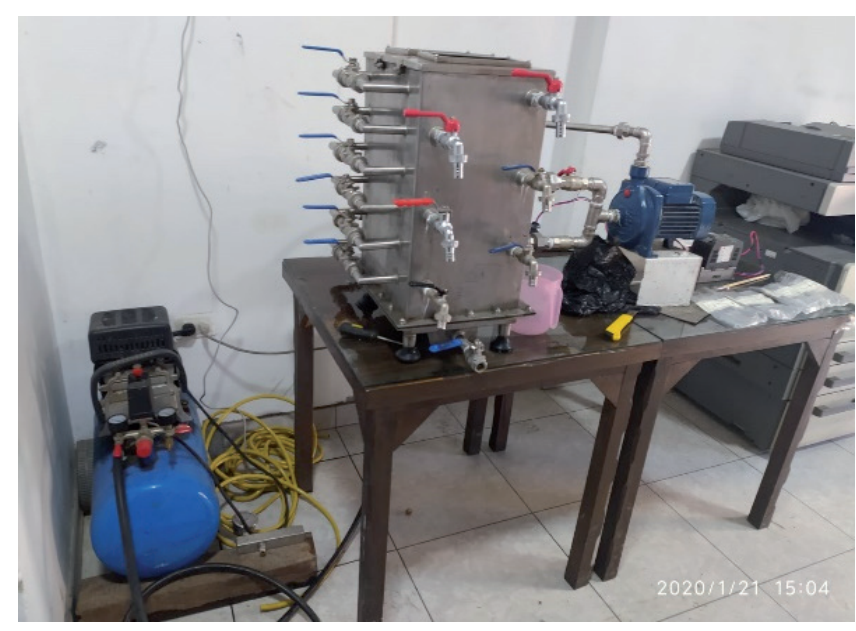

Figura 4.-Equipo de generación de nano burbujas de aire. Fuente: Autorizado por el Dr. Jhonny Valverde

Cada muestra tuvo un tamaño de entre 7 a $10 \mathrm{~cm}$ en los tres lados con evidente presencia de patologías. Todas estas muestras estuvieron expuestas dentro del equipo por 10 minutos, 20 minutos y 30 minutos. De este modo se evidenció que cada muestra presentaba diferentes resultados con distintas cantidades de tiempo de aplicación, las que fueron comparadas entre sí.

-Procedimiento 4: Registro de evaluación de resultados inmediatos

Después de la aplicación de nano burbujas de aire, se realizó un registro fotográfico a cada uno de los ejemplares para identificar si surgían cambios instantáneos. Se efectuó también un proceso de observación a estos testigos para poder reconocer la reacción a la aplicación de nano burbujas de aire [figura 5].

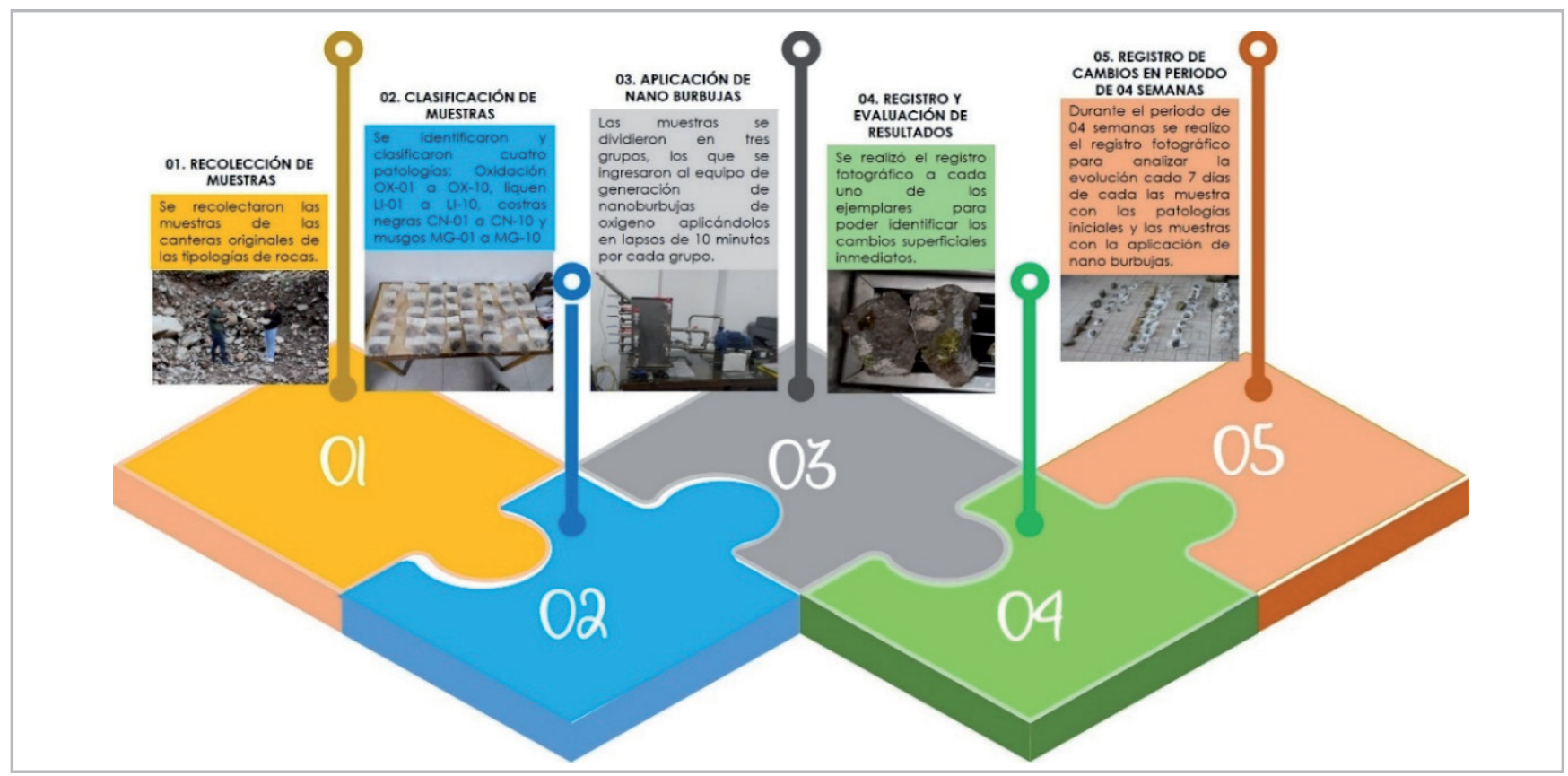

Figura 3.-Procedimiento experimental de aplicación de nanoburbujas de aire. Fuente: propia 


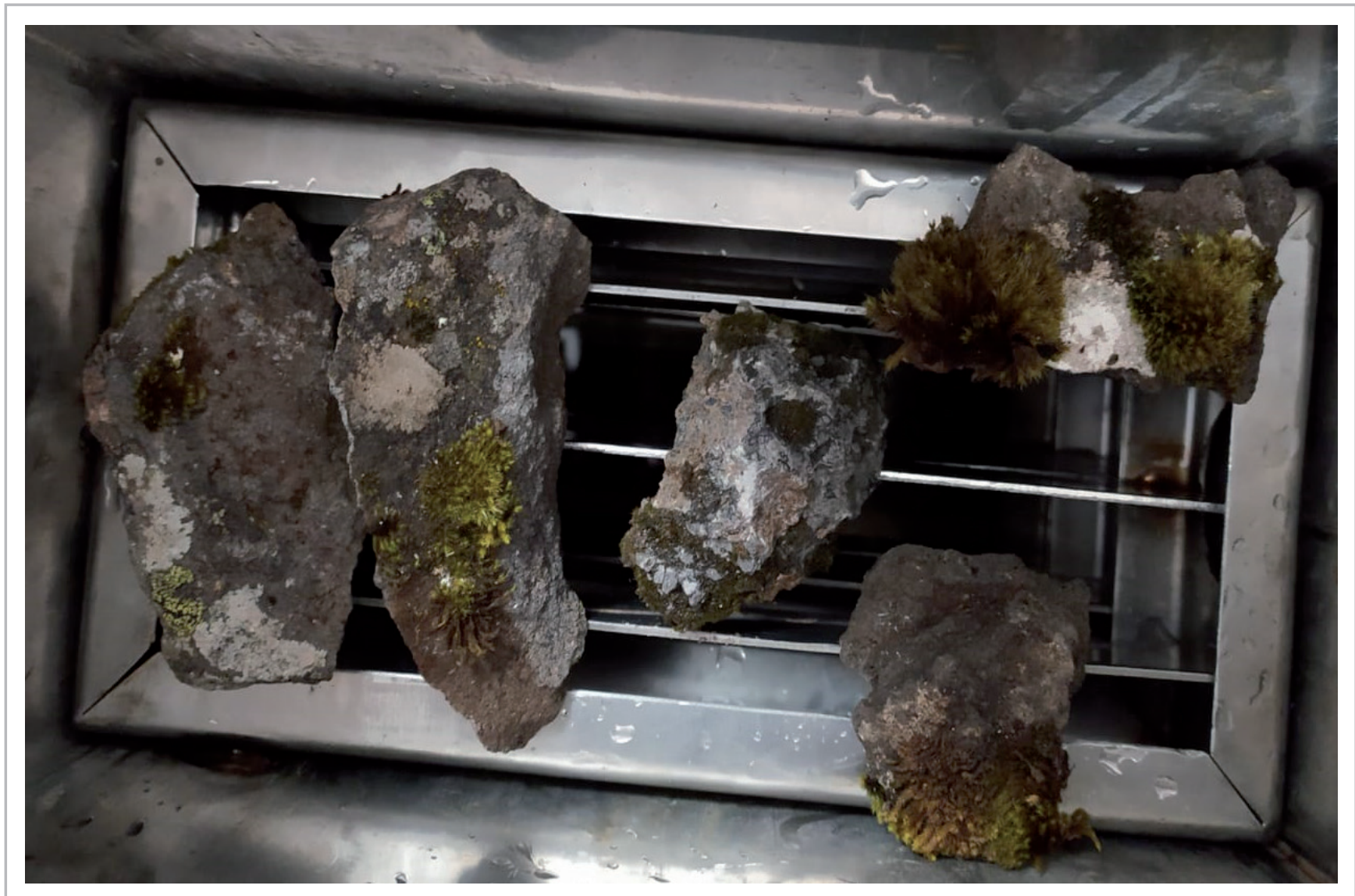

Figura 5.-Muestras en el interior del equipo de nanoburbujas antes y durante el procedimiento. Fuente: propia

-Procedimiento 5: Registro de cambios en periodo de 04 semanas

Una vez aplicadas las nanoburbujas de aire, se observaron los cambios en las muestras a lo largo de 4 semanas en las que estuvieron expuestas tanto las muestras iniciales como la muestra control, a los mismos factores climatológicos, contaminantes, humedad, asoleamiento siendo controladas cada 7 días y registrando fotográficamente los cambios en dichas muestras para establecer si existía efectos posteriores al tratamiento con nanoburbujas de aire.

\section{Resultados}

Los resultados fueron divididos según cada patología, ya que cada una de ellas respondió de modo diferente a la aplicación de nanoburbujas de aire.

\section{Resultados en los agentes bióticos: líquenes}

La aplicación de nanoburbujas de aire para las patologías bióticas arrojan tres resultados diferentes en cada tiempo de exposición, así tenemos que todas las muestras a los diez minutos de aplicación no muestran cambio en sus características superficiales observándose el color aún más verdoso en los líquenes y en las siguientes semanas de observación se manifiesta crecimiento mayor a la muestra inicial. Sin embargo, en las muestras con exposición de veinte minutos, se observa la reducción inmediata y semanas después la patología manifiesta el secado del liquen y disminución en sus dimensiones. Finalmente, en el caso de las muestras de treinta minutos, los cambios se evidencian a lo largo de las cuatro semanas apreciándose el secado íntegro de la patología, así como su desprendimiento al $100 \%$.

\section{Resultados en los agentes bióticos: musgo}

En el caso del agente biótico - musgo, los resultados son muy similares a los del liquen; ya que las muestras expuestas en la primera etapa de aplicación que fue de diez minutos, la patología muestra un reverdecimiento del musgo y mayor crecimiento en las cuatro semanas que se puso en observación. En las exposiciones de veinte y treinta minutos, el agente biótico si manifiesta resultados positivos, que inmediatamente después de la exposición en esos tiempos, el musgo se desprende de la roca sin evidenciar alteraciones en la misma. Dichos resultados, se manifiestan en mayor medida en exposiciones de treinta minutos que pasado siete días de observación, las muestras presentan el $100 \%$ de desprendimiento y secado del musgo y que, a los veintiún días no manifiesta reaparición de la patología. 
Valverde Flores J. W., Vargas Febres C. G., Achahui Alvarez A. C. Rodriguez Salas D. G.

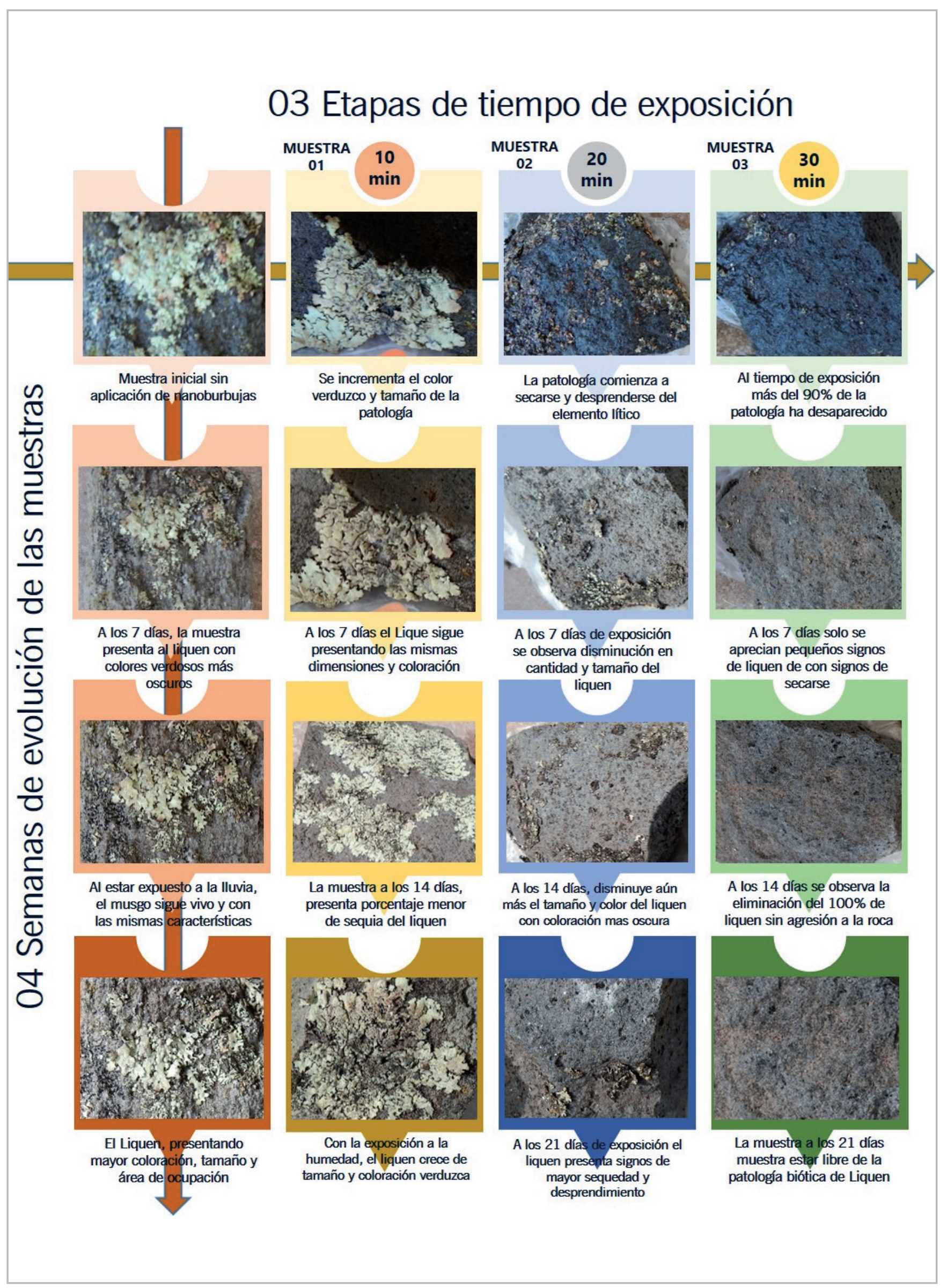

Figura 6.-Resultados de las muestras de patología Líquenes. Fuente: propia 


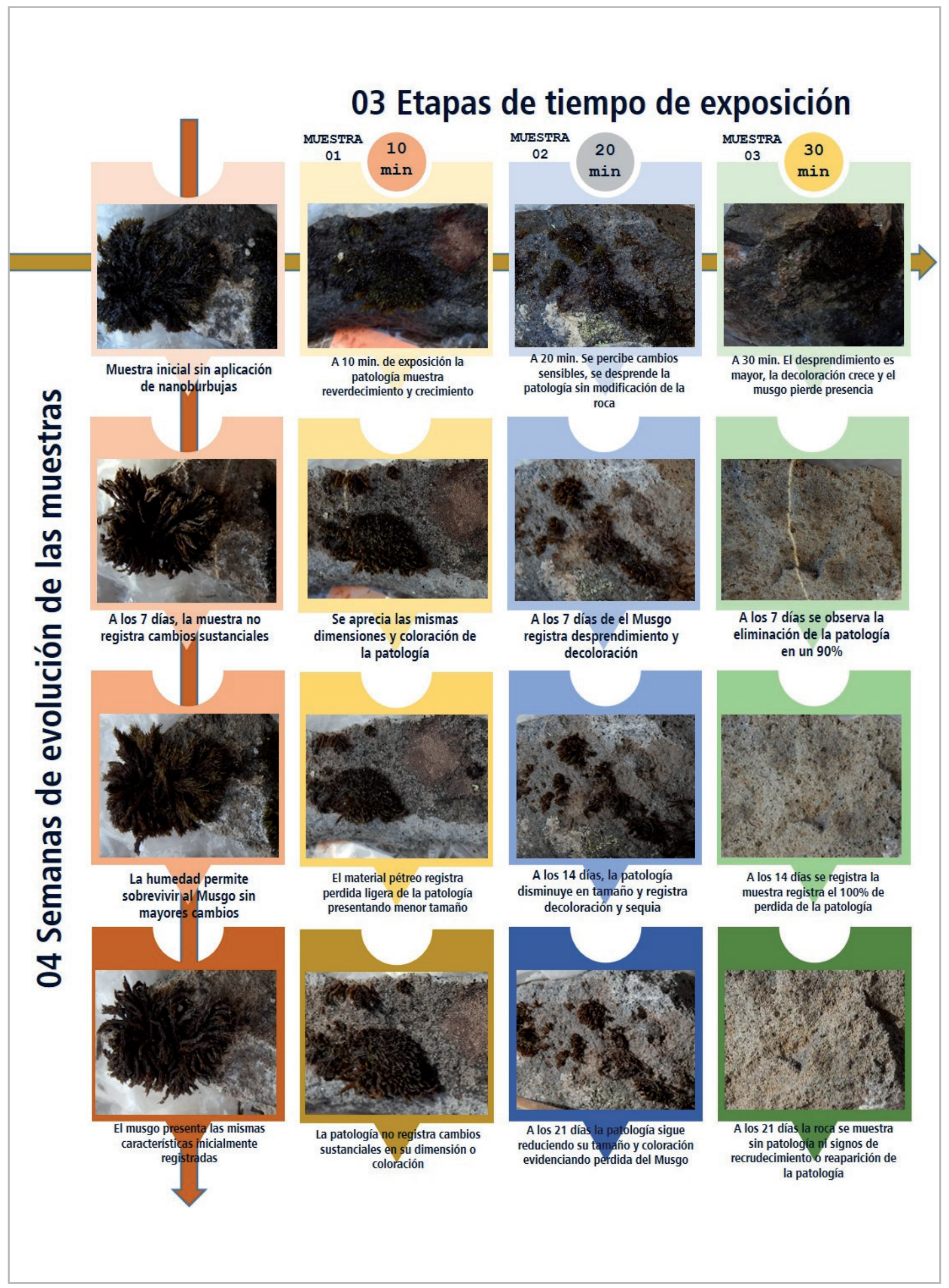

Figura 7.-Resultados de las muestras de patología Musgo. Fuente: propia 


\section{Conclusiones}

Luego de observar y analizar los resultados obtenidos en la experimentación, se concluye:

- En las dos patologías analizadas, a la exposición de nanoburbujas de aire a un lapso de tiempo de diez minutos, no se produce modificaciones superficiales ni desprendimiento o decoloración, presentándose una coloración verdosa y mayor resistencia a un frotado físico. Por tanto, no es recomendable su aplicación en periodos de tiempo menores a 10 minutos ya que a este tiempo refuerza y revive a la patología.

- Las muestras expuestas a un periodo de veinte minutos, manifiestan diferentes respuestas; así los líquenes y musgos, manifiestan sequedad y desprendimientos de la patología, sin manifestar alteraciones evidentes en la estructura de la roca. Se recomienda este tiempo de aplicación para poder retirar parte de estas patologías.

- Los resultados de las muestras a exposición de treinta minutos, si manifiestan cambios en las patologías, los líquenes y musgos presentan un desprendimiento al 100\% evidenciando la limpieza de la patología sin agresión al elemento lítico. Se recomienda este método para la limpieza total del material.

- En conclusión, cantidades en exceso de nanoburbujas de aire desprenden patologías como líquenes y musgos presentes en elementos patrimoniales hechos en piedra. Por lo tanto, es un método económicamente accesible, ecológico y no necesita de mucho tiempo para su aplicación y resultados.

\section{Referencias}

ABATE B., VALVERDE J. (2017). "Reduction of Thermotolerant Coliforms Present in the Sea Water by Means of MicroNanobubbles of Air-Ozone of the Beach Los Pavos", Lima, Peru, Chemical Engineering Transactions (60) 313-318. https://doi. org/10.3303/CET1760053.

ANGLES VARGAS, V. (1988). "Historia del Cusco Incaico". Lima: Industrialgráfica S.A.

ARANCIBIA IGLESIAS, S., ROMEO SÁEZ, A., FERNÁNDEZ MUÑOZ, N. (2015). "Formas de deterioro presentes en las fachadas de piedra de "El Arsenal". Arquitectura y Urbanismo, 79-91.

BROTO COMERMA, C. (2006). “Enciclopedia Broto de las Patologías de la Construcción". Links International.

CABRERA GARRIDO, J. (1979). Causas de alteración y métodos de conservación aplicables a los monumentos hechos con piedra. Materiales cde Construcción, 5-42.

COVARRUBIAS POZO, J. M. (1958). Cuzco Colonial y su Arte. Cusco: $H \& G$ Rozas S. A.
GAINZA LUZEA, M. (2016).“El Deterioro de la piedra en el patrimonio construido y aplicación de nano formulaciones para la conservación de los mismos". Barcelona: universidad politécnica de Cataluña.

GOMEZ VILLALBA, L.S. (2008).“Nanopartículas para la conservación del patrimonio". La conservación de los geomateriales utilizados en el patrimonio, 171-180.

HARING, C. H. (1966). "El imperio Hispánico en América". Editorial Solar/Hachette.

SAMANEZ ARGUMEDO, R. (2019). "Plaza de Armas de Cusco". (A. Achahui, Entrevistador). 15 de diciembre de 2019.

VALENZUELA L., VALVERDE FLORES J. (2018). "Reduction of Lead and Silicon in Wastewater from Gas Scrubbing of a Company using Micronanobubbles of Air-Ozone", Chemical Engineering Transactions (67) 517-522. https://doi.org/10.3303/CET1867087

\section{Autor/es}

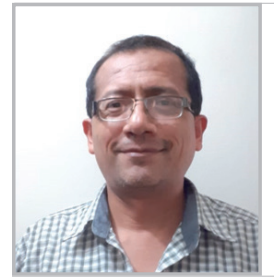

Jhonny Wilfredo Valverde Flores

jvalverde@lamolina.edu.pe Investigador invitado de la Universidad Nacional Agraria la Molina Facultad de Ciencias, Universidad Nacional Agraria la Molina, Lima, Perú

Ingeniero Químico, Maestro en Ingeniería Industrial y Doctor en Ciencias e Ingeniería de la Universidad Nacional de Trujillo. Especialista en Proyectos de Investigación, Inversión y de Desarrollo, Especialista en Análisis de Ciclo de Vida, Especialista en Saneamiento ecológico, Especialista en Gestión Ambiental Rentable, Especialista en Gestión de la Innovación y tecnología y Especialista en Nanotecnología ambiental. Actualmente se dedica a investigar la reducción de parámetros físico-químicos y microbiológicos aplicando nanoburbujas, disminución de contaminación de aguas y energías alternativas aplicando grafeno; así como reducción de metales y bacterias aplicando nanopartículas. Cuenta con patentes y artículos publicados en Scopus.

https://orcid.org/0000-0003-2526-112X

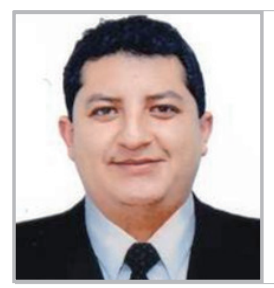

Carlos Guillermo Vargas Febres

cvargasfebres@hotmail.com

Escuela Profesional de Arquitectura, Universidad Andina de Cusco, Peru

Arquitecto de profesión, con estudios de Post grado a nivel de maestría y doctorado, consultor especialista en proyectos de inversión pública en educación y salud, así como del sector privado. Investigador y docente en la escuela profesional de Arquitectura de la Universidad Andina de Cusco.

https://orcid.org/0000-0001-7532-2993 

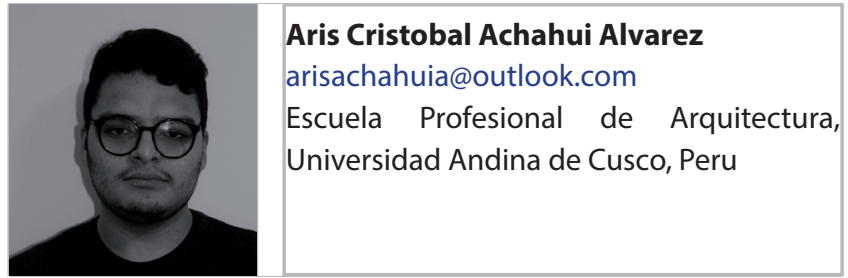

Estudiante de la Escuela Profesional de Arquitectura, Facultad De Ingeniería Y Arquitectura de La Universidad Andina del Cusco, investigador que integra el Centro de Investigación en Arquitectura Tecnología y Urbanismo CIATU -Cusco Perú. https://orcid.org/0000-0001-5692-2707

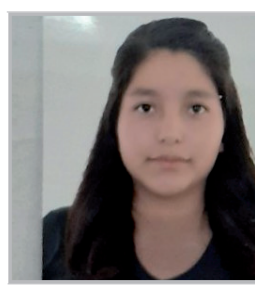

\section{Danna Gabriela Rodriguez Salas}

dannagabrielarosal@gmail.com Escuela Profesional de Arquitectura, Universidad Andina de Cusco, Peru

Estudiante de la Escuela Profesional de Arquitectura, Facultad De Ingeniería Y Arquitectura de La Universidad Andina del Cusco, investigador que integra el Centro de Investigación en Arquitectura Tecnología y Urbanismo CIATU -Cusco Perú.

https://orcid.org/0000-0002-4414-9259

Artículo enviado el 31/08/2020

Artículo aceptado el 25/01/2021

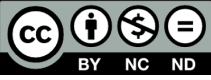

https://doi.org/10.37558/gec.v19i1.812 